\title{
Photon Strength Functions at the Low-Energy Tail of GEDR
}

\author{
Milan Krtička ${ }^{1, a}$ and František Bečvár ${ }^{1}$ \\ Faculty of Mathematics and Physics, Charles University in Prague, 18200 Prague, Czech Republic
}

\begin{abstract}
It is demonstrated that available experimental information on photon strength functions (PSFs) at energies below about $10 \mathrm{MeV}$ is far from desired. Problems that occur during the extraction of PSFs from (n, $\gamma$ ), $\left(\gamma, \gamma^{\prime}\right)$, and ${ }^{3} \mathrm{He}$-induced reactions are discussed.
\end{abstract}

\section{Introduction}

Decay of excited levels at energies above the pairing gap is often described within statistical approach embodying the concept of compound nucleus [1], the validity of Brink hypothesis [2] and the paradigm of photon strength function (PSF) for different types $X$ (electric or magnetic) and multipolarities $L$ of radiation [3]. The photon strength function $f^{(X L)}$ is usually defined via an expectation value of a partial radiation width $\bar{\Gamma}_{a \gamma b}$ for $\gamma$ decay from an initial level $a$ with energy $E_{a}$, spin $J_{a}$ and parity $\pi_{a}$ to a final level $b$ as

$$
\bar{\Gamma}_{a \gamma b}=f^{(X L)}\left(E_{\gamma}\right) E_{\gamma}^{3} / \rho\left(E_{a}, J_{a}, \pi_{a}\right)
$$

Here $\rho(E, J, \pi)$ stands for density of nuclear levels with en$\operatorname{ergy} E$, spin $J$ and parity $\pi$. In line with the Brink hypothesis the $f^{(X L)}$ is dependent only on energy of emitted $\gamma$ radiation and independent of any other properties of the initial and/or final level.

Individual partial radiation widths $\Gamma_{a \gamma b}$ at high energies are believed to be a subject to very strong fluctuations around the expectation value $\bar{\Gamma}_{a \gamma b}$. Assuming that the wavefunctions of highly excited states are very complex, Porter and Thomas [4] showed that the fluctuation properties of $\Gamma_{a \gamma b}$ should be governed by a $\chi^{2}$ distribution with one degree of freedom. This distribution is often called the Porter-Thomas distribution. According to the principle of the detailed balance there is a link between the $f^{(X L)}$ and the energy-smoothed part of the photoabsorption cross section $\bar{\sigma}_{\gamma}^{(X L)}$ for radiation of type $X L$ given by

$$
f^{(X L)}\left(E_{\gamma}\right)=\frac{1}{3(\pi \hbar c)^{2}} \frac{1}{E_{\gamma}} \bar{\sigma}_{\gamma}^{(X L)}\left(E_{\gamma}\right) .
$$

It is well known that $\bar{\sigma}_{\gamma}^{(X L)}$ is dominated by a broad $E 1$ resonance - the giant electric dipole resonance (GEDR). The position of the maximum of the GEDR, can be well described by $31.2 A^{-1 / 3}+20.6 A^{-1 / 6} \mathrm{MeV}$ and its damping width is about $4 \mathrm{MeV}$. A good description of the shape of the resonance near its maximum seems to be given by the

\footnotetext{
a e-mail: krticka@ipnp.troja.mff.cuni.cz
}

Lorentzian curve. Macroscopically, the GEDR is usually described as a vibration of proton versus neutron fluid and it splits into two or three components if the nucleus is axially deformed or triaxial, respectively. The parameters of the Lorentz curve are usually obtained from fitting $(\gamma, \mathrm{xn})$ data near the maximum of the resonance [5].

For many applications it is important to know the behavior of PSFs not only in the vicinity of the GEDR maximum but also at its low-energy tail, especially below neutron separation energy $B_{n}$. Of course, $(\gamma, \mathrm{xn})$ data are unable to answer the question whether the Lorentzian curve gives a reasonable approximation of the $E 1 \mathrm{PSF}$ and whether other types of transitions $X L$ do not play an important role at these energies. It implies that data from other reactions are required.

Main sources of information on $f^{(X L)}$ below $B_{n}$ are data from $(\mathrm{n}, \gamma),\left(\gamma, \gamma^{\prime}\right)$, and ${ }^{3} \mathrm{He}$-induced reactions. As can be seen from Fig. 1, data from these reactions are not always consistent. This paper tries to point out that $f^{(X L)}$ obtained from all these reactions should be taken with care and any of them may be incorrect.

\section{Data from $(\mathbf{n}, \gamma)$ reaction}

There are two main methods how to determine $f^{(X L)}$ from radiative neutron capture: (i) measurements of intensities of primary transitions from isolated resonances or from socalled average-resonance capture [6], and (ii) processing of experimental data from so-called two-step and multi-step $\gamma$ cascades.

\subsection{Primary transitions from $(\mathrm{n}, \gamma)$ reaction}

Intensities of primary transitions from individual resonances or average-resonance capture measurements give "the most direct" information on $f^{(X L)}$ from all the reactions discussed in this contribution. As evident from Eq. (1), there are two quantities needed for determination of $f^{(X L)}$. Namely, the partial radiation widths of primary transitions (that can be obtained by proper normalization of measured intensities 


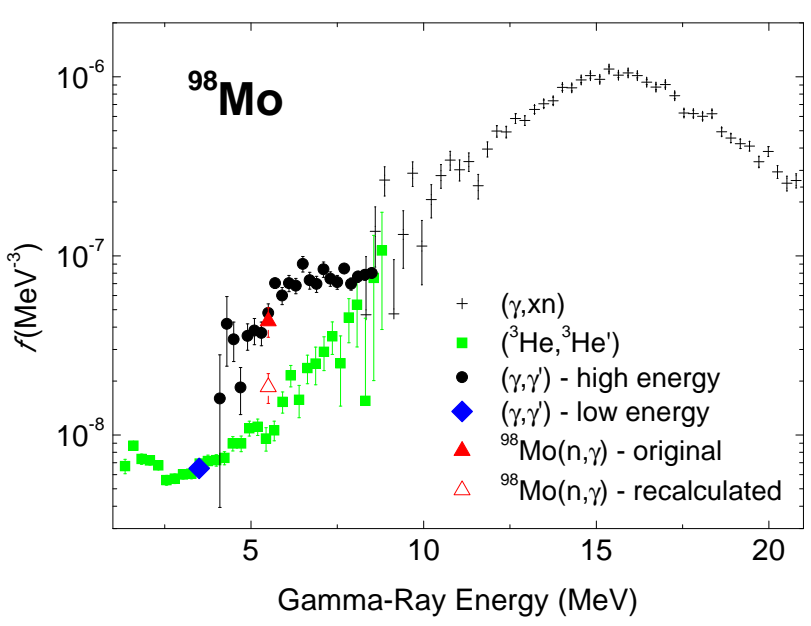

Fig. 1. Available data on PSFs in ${ }^{98} \mathrm{Mo}$. Data taken from from [10] - photonucluear data, [8,9] - $\left(\gamma, \gamma^{\prime}\right)$, and [7] - $\left({ }^{3} \mathrm{He},{ }^{3} \mathrm{He}{ }^{\prime}\right)$ reactions. In addition, data from intensities of primary transitions following radiative neutron capture in ${ }^{98} \mathrm{Mo}$ [11] are shown. For recalculated values see Tab. 1 .

of $\gamma$ rays) and level density of states with given $J$ and $\pi$ at neutron resonances. Supposing that the partial radiation widths are determined correctly there is still an uncertainty coming from level density. Densities of neutron resonances are determined using time-of-flight measurements in which all the stronger resonances are usually observed. If the number of observed resonances is sufficiently high one can deduce density near $B_{n}$ with relatively high precision. On the other hand, if density used in estimation of $f^{(X L)}$ is based only on a few resonances the value can be significantly biased.

It should be also stressed that due to Porter-Thomas fluctuations of reduced neutron widths it is almost impossible to observe all the resonances as some of them are very week. Different systematics of level densities try to bypass this problem using different approaches for correcting experimental data on unobserved resonances. But unbiased correction is very difficult, especially in cases where not many resonances are observed.

The systematics of $f^{(X L)}$ from primary transitions was prepared by Kopecky [11]. Level densities at neutron separation energy used in this systematics are available which makes recalculation of $f^{(X L)}$ using newer information on level density possible. Recalculated values of $f^{(X L)}$ may significantly differ from original ones as shown in Tab. 1 for nuclei with $90<A<100$. Here, the original values of $f^{(X L)}$ [11] are compared with those obtained with help of level densities taken from Ref. [12]. Recalculated value for ${ }^{99} \mathrm{Mo}$ is plotted also in Fig. 1. Of course, we are unable to say which of the two level densities are closer to reality but only to show that significant uncertainty on $f^{(X L)}$ comes from not exact knowledge of level density.

\subsection{Two- and multi-step $\gamma$ cascades}

Data from so-called two-step cascade (TSC) method [13] become a source of interesting information on $f^{(X L)}$ dur- ing last 15 years. In TSC experiment, the $\gamma$-ray spectra belonging to all TSCs connecting the initial level arising from thermal neutron capture with preselected low-lying levels in the product nuclei are accumulated using two Ge detectors, see e.g. $[14,15]$. The method does not allow direct determination of $f^{(X L)}$ but it requires comparison of experimental spectra with results of simulations based on different assumptions on $f^{(X L)}$ and level density. Simulations are usually performed with help of the computer code DICEBOX [16]. Recently, similar approach, i.e. comparison of experimental spectra with DICEBOX simulations, was applied also to measurement of so-called multi-step $\gamma$ cascades (MSC) following neutron capture on isolated resonances [17]. Highly segmented $4 \pi \mathrm{BaF}_{2}$ detektor was used in these measurements. Photon strength functions deduced from these experiments are not shown in Fig. 1 as they are not available for ${ }^{98} \mathrm{Mo}$.

Simulations of TSC and MSC spectra are sensitive to energy dependence of $f^{(X L)}$ and to ratios of PSFs for different types $X L$. But they are insensitive to absolute values of $f^{(X L)}$. The only quantity simulated by the DICEBOX code which is sensitive to absolute values of $f^{(X L)}$ is the total radiation width of neutron resonances

$$
\Gamma_{a \gamma}=\sum_{b, E_{b}<E_{a}} \Gamma_{a \gamma b}
$$

This "integral" quantity is sensitive not only to values of $f^{(X L)}$ but also to level level in the whole region below $B_{n}$ and might be used for normalization of tested models. Instead of normalizing tested models to $\Gamma_{a \gamma}$ this quantity we usually use it as an independent test of correctness of models used in simulations.

\section{Data from ${ }^{3} \mathrm{He}$-induced reactions}

Rich information on $f\left(E_{\gamma}\right)=\sum_{X L} f^{(X L)}\left(E_{\gamma}\right)$ was obtained from measurements of $\left({ }^{3} \mathrm{He},{ }^{3} \mathrm{He} \gamma\right)$ and $\left({ }^{3} \mathrm{He}, \alpha \gamma\right)$ reactions on the Oslo cyclotron during past 15 years, see e.g. [7,18]. For each initial excitation energy $E_{a}$, determined from the ejectile energy, $\gamma$-ray spectra are recorded in this kind of experiments. Recorded $\gamma$ spectra are unfolded using the known $\gamma$-ray response function of the detector set up. Socalled first-generation (or primary) $\gamma$-ray matrix $P\left(E_{a}, E_{\gamma}\right)$ is constructed from these unfolded spectra. The matrix is then factorized according to the Brink-Axel hypothesis

$$
P\left(E_{a}, E_{\gamma}\right) \propto \rho\left(E_{a}-E_{\gamma}\right) \times 2 \pi E_{\gamma}^{3} f\left(E_{\gamma}\right) .
$$

Functions $\rho\left(E_{b}\right)$ and $f\left(E_{\gamma}\right)$ can be determined by an iterative procedure [19]. But decomposition of $P\left(E_{a}, E_{\gamma}\right)$ into $\rho\left(E_{b}\right)$ and $f\left(E_{\gamma}\right)$ is not unique. If one solution is known, one may construct an infinite number of other functions, which give identical fits to the $P$ matrix by

$$
\begin{aligned}
\tilde{f}\left(E_{\gamma}\right) & =N_{f} e^{-\alpha E_{\gamma}} f\left(E_{\gamma}\right) \\
\tilde{\rho}\left(E_{a}-E_{\gamma}\right) & =N_{\rho} e^{\alpha\left(E_{a}-E_{\gamma}\right)} \rho\left(E_{a}-E_{\gamma}\right) .
\end{aligned}
$$

Here, $N_{f}$ and $N_{\rho}$ are only normalization constants. These constants, together with the slope $\alpha$, are obtained by adjusting functions $\rho\left(E_{b}\right)$ and $f\left(E_{\gamma}\right)$ to data from other experiments. Specifically, $N_{\rho}$ and $\alpha$ are obtained by normalizing 


\begin{tabular}{|c|c|c|c|c|c|c|}
\hline \multirow[t]{2}{*}{ Nucleus } & \multirow[t]{2}{*}{$X L$} & \multirow{2}{*}{$\begin{array}{c}E_{\gamma} \\
(\mathrm{MeV})\end{array}$} & \multicolumn{2}{|c|}{ original [11] } & \multicolumn{2}{|c|}{ recalculated [12] } \\
\hline & & & $\begin{array}{l}\langle D\rangle \\
(\mathrm{eV})\end{array}$ & $\begin{array}{c}f^{(X L)} \times 10^{-8} \\
\left(\mathrm{MeV}^{-3}\right)\end{array}$ & $\begin{array}{l}\langle D\rangle \\
(\mathrm{eV})\end{array}$ & $\begin{array}{c}f^{(X L)} \times 10^{-8} \\
\left(\mathrm{MeV}^{-3}\right)\end{array}$ \\
\hline \multirow[t]{2}{*}{${ }^{93} \mathrm{Mo}$} & $E 1$ & 6.6 & 1000 & 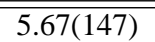 & $2700(500)$ & $2.10(54)$ \\
\hline & $M 1$ & 6.2 & & $1.46(42)$ & & $0.54(16)$ \\
\hline${ }^{95} \mathrm{Mo}$ & $E 1$ & 7.3 & 975 & $5.38(41)$ & $1320(180)$ & $3.97(30)$ \\
\hline \multirow[t]{2}{*}{${ }^{99} \mathrm{Mo}$} & $E 1$ & 5.5 & 429 & $4.32(81)$ & $1000(200)$ & $1.85(35)$ \\
\hline & $M 1$ & 5.5 & & $0.59(18)$ & & $0.25(7)$ \\
\hline \multirow[t]{2}{*}{${ }^{94} \mathrm{Nb}$} & $E 1$ & 6.5 & 37.8 & $5.04(124)$ & $80(10)$ & $2.38(59)$ \\
\hline & $M 1$ & 6.5 & & $1.20(44)$ & & $0.57(21)$ \\
\hline
\end{tabular}

Table 1. Photon strength functions deduced from intensities of primary transitions in $(\mathrm{n}, \gamma)$ reactions. Recalculated values were obtained using resonance spacings taken from [12]. No error was added to $f^{(X L)}$ due to uncertainty in resonance spacings listed in [12].

the level density to the number of known discrete levels at low excitation energy and to the level density estimated from neutron-resonance spacing at $B_{n}$. Factor $N_{f}$ is then usually obtained to utilize experimental data on $\Gamma_{a \gamma}$.

Supposing the functions $\rho\left(E_{b}\right)$ and $f\left(E_{\gamma}\right)$ are obtained correctly the determination of $\alpha$ and normalization factors may be a source of problems as they depend on the density of neutron resonances. In addition to the problem with the level density near $B_{n}$ mentioned in Sec. 2.1 a new problem occurs here. Level density measured in Oslo experiments is very probably close to the total level density, i.e. to the sum of densities for all spins and parities. But spacing of neutron resonances gives information only on level density for one or two spins. Recalculation of resonance data to total level density is usually done via relation

$$
\rho(J)=\frac{2 J+1}{2 \sigma_{c}^{2}} \exp \left(-\frac{(J+1 / 2)^{2}}{2 \sigma_{c}^{2}}\right)
$$

where $\sigma_{c}$ is spin-cut off parameter. Unfortunately, the value of $\sigma_{c}$ is not known with sufficient precision which can lead to biased estimates of $f\left(E_{\gamma}\right)$ and $\rho\left(E_{b}\right)$. Illustration of the influence of the different $\sigma_{c}$ on the results is shown in Fig. 2. Validity of Eq. (7) is questionable too.

In addition to problems with normalization of $\rho\left(E_{b}\right)$ and $f\left(E_{\gamma}\right)$ there is a question whether the factorization of matrix $P\left(E_{a}, E_{\gamma}\right)$ according to Eq. (4) can be done. It is very difficult to estimate how the results were related to reality if the function $f\left(E_{\gamma}\right)$ were not dependent only on $E_{\gamma}$ but also on the excitation energy.

\section{Data from $\left(\gamma, \gamma^{\prime}\right)$ reactions}

Data from $\left(\gamma, \gamma^{\prime}\right)$ reaction can yield information on $f\left(E_{\gamma}\right)$ that corresponds only to ground state transitions. A series of $\left(\gamma, \gamma^{\prime}\right)$ measurements with the aim to learn about $f\left(E_{\gamma}\right)$ at energies above about $4 \mathrm{MeV}$ was performed at the Forschungszentrum Dresden with a beam of photons produced by bremsstrahlung, see e.g. [9]. The beam covers all $\gamma$ energies up to about $13 \mathrm{MeV}$. As levels at higher excitation energies decay not only to the ground state but also to excited states the measured spectrum of outgoing $\gamma$-rays becomes very complicated. This spectrum does not contain only photons connecting the level excited directly
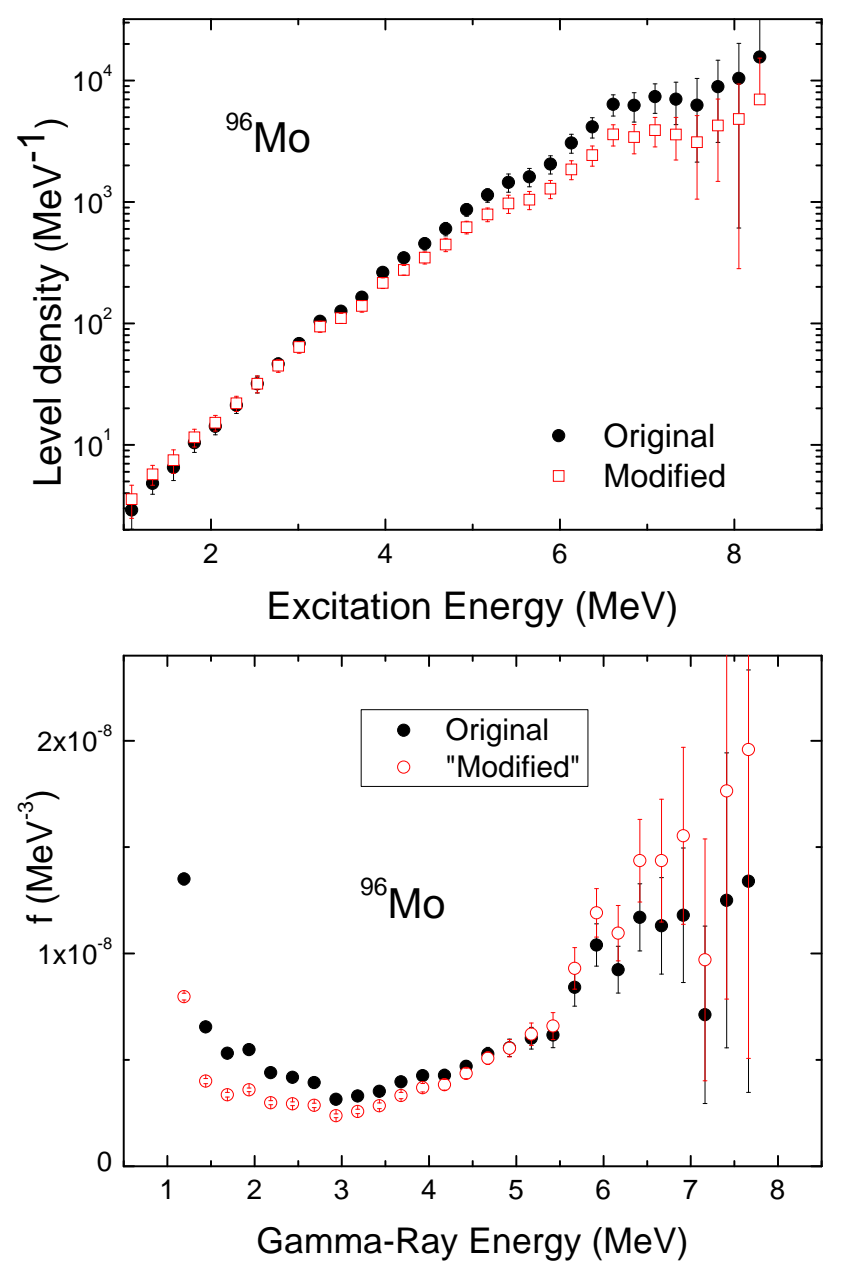

Fig. 2. Illustration of possible impact of different values of $\sigma_{c}$ on deduced experimental results from ${ }^{3} \mathrm{He}$-induced reactions. Upper figer - total level density, lower figure - PSF. "Original" data correspond to those published[7] while the "modified" ones to an artificial situation when the contribution of levels with $J=$ $1 / 2$ with respect to all levels is one half of the value assumed in [7]. Such a situation happens, for instance, when the spin cut-off parameter is changed from $\sigma_{c}=3$ to $\sigma_{c}=4$. 
by incoming photon with the ground state but also many transitions between excited states. It means that extraction of $f\left(E_{\gamma}\right)$ from experimental data requires analysis of the spectrum with help of auxiliary simulations. In fact, neglecting contributions of $\gamma$ rays from excitation energies above $B_{n}$ the spectrum of energies just below $B_{n}$ is free of any contamination and allows direct determination of $f\left(E_{\gamma}\right)$.

In Ref. [9] the analysis of the spectrum started at energy just below $B_{n}$ and the contribution from $\gamma$ rays corresponding to decays of levels at this excitation energy to all other excited states was estimated and subtracted using DICEBOX code. In the next step, energies at slightly lower energies were treated in the same way and the procedure was repeated for lower and lower energies down to about $4 \mathrm{MeV}$. A consistency of obtained results with functions used in simulations was checked at the end of the procedure. Results shown in Fig. 1 were obtained. Evidently, the main problem of such a procedure is that it is not easy to keep all the errors under control especially at lower energies.

In order to check the rigidity of the method we recently tried to adopt different approach. Efficiency corrected experimental spectra were compared with spectra coming directly from DICEBOX simulations. Using the model of $f\left(E_{\gamma}\right)$ shown in the upper part of Fig. 3 the results presented in the lower part of the Figure were obtained. The uncertainties in simulations come from correct treatment of Porter-Thomas fluctuation of $\Gamma_{a \gamma b}$. Shapes of experimental and simulated spectra seem to be reasonably consistent here. As the function used in simulations differed significantly from results obtained by the procedure applied in Ref. [9] the figure might indicate that the decomposition of the spectrum of outgoing photons from $\left(\gamma, \gamma^{\prime}\right)$ measurements is not unique. Unfortunately, no additional tests have been made and this phenomenon requires further study.

\section{Conclusions}

On the example of molybdenum isotopes it was shown that the present experimental information on photon strength functions is far from desired. Problems occurring in determination of PSFs from (n, $\gamma),\left(\gamma, \gamma^{\prime}\right)$, and ${ }^{3} \mathrm{He}$-induced reactions were addressed. It can be concluded that PSFs obtained from all these reactions must be taken with care and further studies are required to reach the final goal consistent PSFs from all experiments.

This work was supported by the research plans MSM 0021620859 and INGO LA08015 of the Ministry of Education of the Czech Republic.

\section{References}

1. N. Bohr, Nature 137 (1936) 344

2. D.M. Brink, PhD Thesis, Oxford University (1955)

3. G.A. Bartholomew, Ann. Rev. Nucl. Sci. 11 (1961) 259
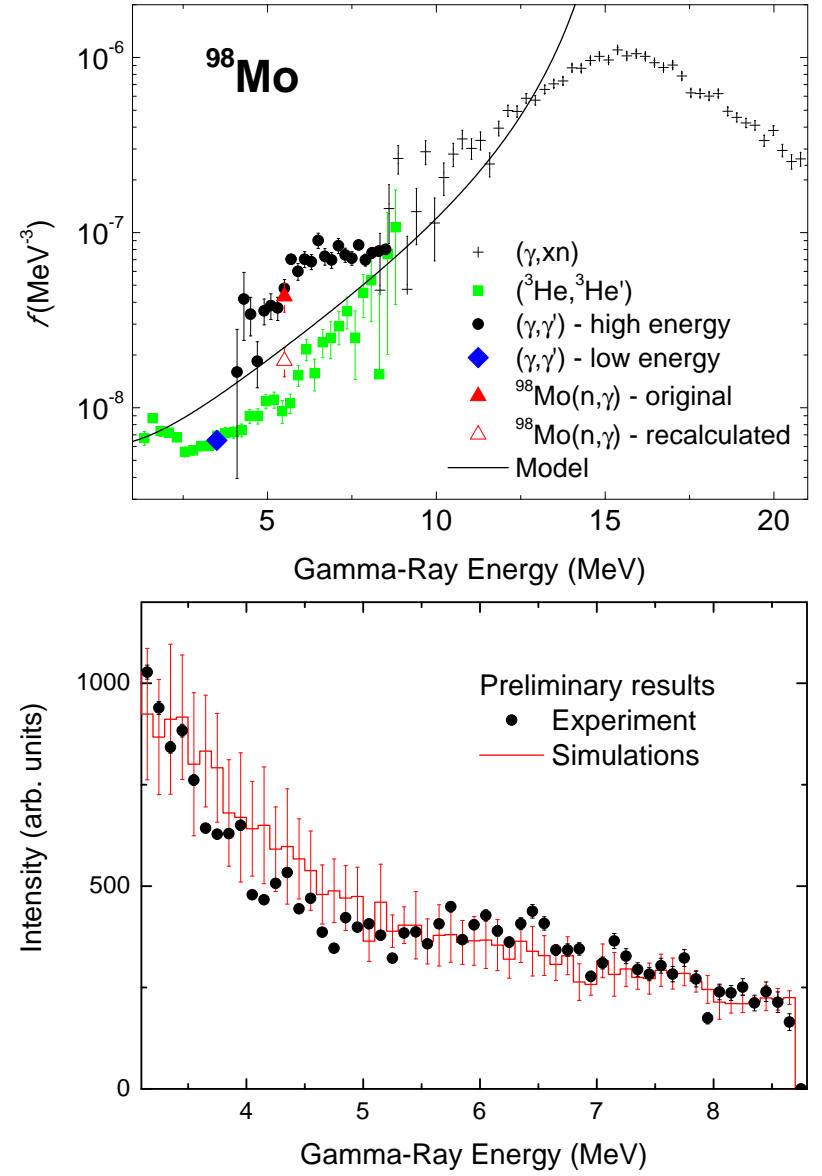

Fig. 3. Comparison of efficiency-corrected experimental spectra with results of DICEBOX simulations (lower part of the figure). Simulations were made with model of PSF shown in the upper part of the figure. Data are the same as in Fig. 1.

4. C.E. Porter and R.G. Thomas, Phys. Rev. C 2 (1956) 2030

5. S.S. Dietrich and B.L. Berman, At. Data and Nucl. Data Tables 38 (1988) 199

6. L.M. Bollinger and G.E. Thomas, Phys. Rev. C2 (1970) 1951

7. M. Guttormsen et al., Phys. Rev. C 71, 044307 (2005)

8. G. Rusev et al., Phys. Rev. C 73, 044308 (2006)

9. G. Rusev et al., Phys. Rev. C 77, 064321 (2008)

10. H. Beil et al., Nucl. Phys. A 227, 427 (1974)

11. J. Kopecky, M. Uhl, contribution to the NEA/ENEA and IAEA specialists' meating on measurement, calculation and evaluation of photon production data, Bologna, Italy, Nov. 9-17, 1994

12. T. Belgya et al., RIPL2; available online at http://www-nds.iaea.org/RIPL-2/, IAEA, Vienna, 2005.

13. J. Honzátko et al., Nucl. Instr. Meth. A 376 (1996) 434

14. L. Zanini et al, Phys. Rev. C 68 (2003) 14320

15. M. Krticka et al., Phys. Rev C 77, 054319 (2008)

16. F. Bečvář, Nucl. Instr. Meth. A 417, (1998) 434

17. S.A. Sheets, Phys. Rev C 79, 024301 (2009)

18. contributions to this volume by S. Siem, A.C.Larsen, A. Bürger and M. Guttormsen

19. A. Schiller et al., Nucl. Instr. Meth. A 447 (2000) 498 